\title{
Indirect Detection of Infinite-Speed MAS Solid-State NMR Spectra
}

Frédéric A. Perras, ${ }^{\mathrm{a}}$ Amrit Venkatesh, ${ }^{\mathrm{ab}}$ Michael P. Hanrahan, ${ }^{\mathrm{ab}}$ Tian Wei Goh, ${ }^{\mathrm{b}}$ Wenyu Huang, ${ }^{\mathrm{ab}}$ Aaron J. Rossini, ${ }^{a b}$ and Marek Pruski* ${ }^{a b}$

${ }^{a} U S$ DOE, Ames Laboratory, Ames, IA, 50011, USA

${ }^{\mathrm{b}}$ Department of Chemistry, Iowa State University, Ames, IA, 50011, USA.

* Author to whom correspondence should be addressed

M. Pruski: Ames Laboratory, Iowa State University, 230 Spedding Hall, Ames, IA 50011-3020, USA. Phone: +1 5152942017 Fax: +1 515294 4709. E-mail address: mpruski@iastate.edu.

\begin{abstract}
Heavy spin-1/2 nuclides are known to possess very large chemical shift anisotropies that can challenge even the most advanced magic-angle-spinning (MAS) techniques. Wide manifolds of overlapping spinning sidebands and insufficient excitation bandwidths often obfuscate meaningful spectral information and force the use of static, low-resolution solid-state (SS)NMR methods for the characterization of materials. To address these issues, we have merged fastmagic-angle-turning (MAT) and dipolar heteronuclear multiple-quantum coherence (D-HMQC) experiments to obtain D-HMQC-MAT pulse sequences which enable the rapid acquisition of 2D SSNMR spectra that correlate isotropic ${ }^{1} \mathrm{H}$ chemical shifts to the indirectly detected isotropic "infinite-MAS" spectra of heavy spin-1/2 nuclides. For these nuclides, the combination of fast MAS and ${ }^{1} \mathrm{H}$ detection provides a high sensitivity, which rivals the DNP-enhanced ultra-wideline SSNMR. The new pulse sequences were used to determine the Pt coordination environments in a complex mixture of decomposition products of transplatin and in a metal-organic framework with Pt ions coordinated to the linker ligands.
\end{abstract}

\section{Keywords}

Ultra-fast MAS, magic-angle-turning, D-HMQC, platinum NMR, metal-organic-framework

(C) 2017. This manuscript version is made available under the Elsevier user license http://www.elsevier.com/open-access/userlicense/1.0/ 


\section{Introduction}

Solid-state nuclear magnetic resonance (SSNMR) spectroscopy has evolved into one of the premier structural characterization techniques used in biology, chemistry and materials science. While the acquisition and interpretation of SSNMR data in biological systems, as well as many classes of compounds and materials, is now relatively straightforward, there are many circumstances that limit the utility of SSNMR. One such situation is the so-called ultra-wideline regime. $^{1}$

Many heavy spin-1/2 nuclei, namely: ${ }^{119} \mathrm{Sn},{ }^{125} \mathrm{Te},{ }^{195} \mathrm{Pt},{ }^{199} \mathrm{Hg},{ }^{203 / 205} \mathrm{Tl}$, and ${ }^{207} \mathrm{~Pb}$, as well as most quadrupolar nuclei, possess static SSNMR linewidths that exceed the currentlyachievable magic-angle-spinning (MAS) frequencies and the bandwidths of conventional SSNMR probes. For these nuclides, the acquisition of MAS SSNMR spectra is often challenging and counterproductive since spectral overlap of the centerbands and sidebands of the various sites can complicate otherwise simple spectra. ${ }^{2}$ Consequently, the vast majority of methodological developments aimed at addressing these nuclides have focused on facilitating the acquisition of ultra-wideline static SSNMR spectra.

The first major advance in this field was provided by Cheng and Ellis, ${ }^{3}$ as well as Berr and Jones, ${ }^{4}$ who applied a Carr-Purcell-Meiboom-Gill (CPMG) echo train to compress the intensity of broad SSNMR signals into series of sharp spikelets that mimic the static SSNMR lineshapes. This was later further developed into a method of choice by Larsen and coworkers. ${ }^{5,6}$ In spin systems where the effective refocused transverse dephasing time $\left(T_{2}\right)$ is much longer than the transverse relaxation time $\left(T_{2}^{*}\right)$ determined by the observed linewidth, the use of CPMG refocusing can provide a crucial improvement in sensitivity. However, in order to uniformly excite ultra-wideline powder patterns with bandwidths exceeding that of the spectrometer, a 
series of sub-spectra need to be acquired at evenly spaced frequency offsets using an approach termed the variable offset cumulative spectrum (VOCS) acquisition. ${ }^{2,7}$ Persons and Harbison demonstrated that limited spectrometer bandwidth could be overcome with slow sample spinning 2D SSNMR experiments that correlate the resonance frequency at different rotor phases and artificially enhance the excitation bandwidth. ${ }^{8}$ They applied this method, which they named slow-turning reveals enormous anisotropic quadrupolar interactions (STREAQI), exclusively to quadrupolar nuclei. More recently, Bhattacharyya and Frydman demonstrated that frequencyswept wideband, uniform rate and smooth truncation (WURST) shaped pulses could be used to dramatically improve the bandwidth of static spin echo experiments. ${ }^{9,10}$ This concept was then combined with CPMG sensitivity improvement by O'Dell and Schurko, in the WURST-CPMG experiment (WCPMG). ${ }^{11}$ Subsequently, Harris and coworkers designed the related broadband adiabatic inversion cross-polarization (BRAIN-CP) experiment that enables the polarization of broad lines from ${ }^{1} \mathrm{H}$ magnetization. ${ }^{12}$ This technique was further sensitized by us with the addition of dynamic nuclear polarization (DNP). ${ }^{13,14}$

The development of MAS probes capable of spinning samples of up to $130 \mathrm{kHz}{ }^{15}$ opens new prospects for ultra-wideline SSNMR. Faster spinning enables a greater separation of the spinning sidebands, which improves the resolution, ${ }^{16}$ and the use of microcoils can allow the application of very high radio-frequency (rf) field strengths, thus enhancing the excitation bandwidth; indeed rf field strengths as high as $4.7 \mathrm{MHz}$ have been reported. ${ }^{17,18,19}$ These advances have enabled $\mathrm{Hu}$ and co-workers ${ }^{20,21}$ to acquire quantitative, sideband-free, SSNMR spectra of heavy spin-1/2 nuclei by means of magic-angle-turning (MAT) ${ }^{22}$ at a MAS frequency of $22 \mathrm{kHz}$. Hung and coworkers later used the related projection MAT-PASS experiment to 
obtain isotropic ${ }^{7} \mathrm{Li}$ spectra of paramagnetic materials whose linewidths are on the order of 1 $\mathrm{MHz}^{23}$

Unfortunately, the use of small rotors imposes a sensitivity penalty, associated with radically smaller sample size. ${ }^{15}$ To offset this loss, Rossini and co-workers have used a constanttime version of the dipolar-heteronuclear-multiple-quantum-coherence experiment (CT-DHMQC) to enable the acquisition of ultra-wideline SSNMR spectra by ${ }^{1} \mathrm{H}$ detection at ultra-fast MAS rates. ${ }^{24}{ }^{1} \mathrm{H}$ detection is a commonly-used approach to improve the sensitivity of fast MAS experiments, in which the fast sample spinning alleviates the need for homonuclear ${ }^{1} \mathrm{H}-{ }^{1} \mathrm{H} \mathrm{rf}$ decoupling. ${ }^{25-28}$ Unlike the conventional D-HMQC experiments, ${ }^{29,30}$ for which the spectral window in the indirect dimension is limited by the spinning frequency, the CT-D-HMQC experiment enables the use of arbitrary spectral widths and the measurement of the full sideband pattern.

To maximize both sensitivity and resolution, a technique providing isotropic, sidebandfree, spectra would be ideal. Although there are numerous experimental schemes that have been presented for the removal of spinning sidebands, ${ }^{31}$ MAT is most appropriate in the case of ultrawideline SSNMR spectra. Total suppression of sidebands (TOSS), ${ }^{32}$ phase-adjusted spinningsidebands (PASS), ${ }^{33}$ and sideband elimination by temporary interruption of the chemical shift $(\text { SELTICS })^{34}$ all require the application of narrow-banded $\pi$ or spin-locking pulses. Furthermore, these methods suppress the spinning sidebands and thus lead to a considerable loss of intensity. ${ }^{31}$ Contrary to this, MAT condenses all the intensity into the centerband and can be applied in a broadbanded fashion with the application of high-power $\theta$ pulses, ${ }^{20,21}$ or by using short highpower adiabatic pulses (SHAPs). ${ }^{35}$ The MAT experiment making use of SHAPs has been termed adiabatic-MAT (aMAT). ${ }^{36}$ Like the CT-D-HMQC experiment, MAT is also a constant 
time experiment. The successful incorporation MAT in an indirectly-detected D-HMQC experiment could then lead to large improvements in sensitivity and resolution over the CT-DHMQC experiment and enable, for the first time, the acquisition of purely isotropic SSNMR spectra of heavy spin-1/2 nuclei by ${ }^{1} \mathrm{H}$ detection.

\section{Results and Discussion}

\section{Pulse Sequences}

The pulse sequence for the CT-D-HMQC experiment is depicted in Figure 1a, along with its coherence transfer pathway diagram. As can be seen, the ${ }^{1} \mathrm{H}$ spins are first excited by a $\pi / 2$ pulse to create transverse ${ }^{1} \mathrm{H}$ magnetization. Heteronuclear, zero-quantum, dipolar recoupling is then applied to reintroduce the dipolar coupling between nearby H-X spin pairs and create twospin antiphase magnetization that can be subsequently converted into a heteronuclear doublequantum coherence by the application of an excitation pulse on the $\mathrm{X}$ nuclei. ${ }^{37}$ We chose the $\mathrm{SR} 4{ }_{1}^{2}$ recoupling scheme for this task, ${ }^{38}$ because it is less sensitive to $\mathrm{rf}$ inhomogeneity than continuous rotary resonance recoupling $\left(\mathrm{R}^{3}\right)$ irradiation, and in addition provides ${ }^{1} \mathrm{H}$ homonuclear decoupling. ${ }^{39}$ The double-quantum coherence evolves through the sum of the chemical shifts of the ${ }^{1} \mathrm{H}$ and $\mathrm{X}$ spins for a period of $t_{1} / 2$ after which a $\pi$ pulse is applied to refocus the ${ }^{1} \mathrm{H}$ chemical shifts such that the $t_{1}$ period is only encoded by the chemical shifts of the $\mathrm{X}$ nuclei. The pulse sequence is then reversed to convert the heteronuclear double-quantum coherence back into transverse ${ }^{1} \mathrm{H}$ magnetization for detection. The sole application of two short $\theta$ pulses on the $\mathrm{X}$ nuclei ensures the broadbandedness of the method. ${ }^{20,24}$ The total time evolution of the ${ }^{1} \mathrm{H}$ spin echo is kept constant (and rotor-synchronized), to allow the use of arbitrary (i.e., non-rotor-synchronized) increments of $t_{1}$ for better control of the indirect spectral widths. 
The CT-D-HMQC scheme can be easily modified to integrate the MAT experiment ${ }^{22}$ in the indirect dimension. In the resulting D-HMQC-MAT scheme (Figure 1b), the $t_{1}$ evolution period is separated into three equal periods of $t_{1} / 3$, that are timed such that each evolution period is centered on rotor orientations that are $120^{\circ}$ out of phase. As in all MAT schemes, the X magnetization evolves under its $\delta_{\mathrm{xx}}, \delta_{\mathrm{yy}}$, and $\delta_{\mathrm{zz}}$ tensor components for $1 / 3$ of the evolution period, which refocuses the chemical shift anisotropy $(\mathrm{CSA})\left(\delta_{\mathrm{iso}}=1 / 3\left(\delta_{\mathrm{xx}}+\delta_{\mathrm{yy}}+\delta_{\mathrm{zz}}\right)\right)$ and leads to a purely isotropic evolution in $t_{1}{ }^{40}$ The total echo time $(n)$ is again kept constant, noting that $n$ cannot be a multiple of 3 . The desired coherence transfer pathways are selected by nesting the $\operatorname{COG} 3(2,1 ; 0)$ and COG6 $(0,5,0,5,0,5 ; 3)$ cogwheel phase cycles ${ }^{41}$ in the ${ }^{1} \mathrm{H}$ and $\mathrm{X}$ channels, respectively, for a total phase cycle of 18 steps. The cogwheel phase cycles were calculated using Jerschow's CCCP++ software. ${ }^{42}$ An optional 2-phase cycling of the z-filtering pulses can also be applied to improve the coherence selection for a total of 144 steps.

Although it is conceptually easy to implement the fast-MAT experiment in a D-HMQC experiment, the application of three z-filters with short tip angle pulses can lessen MAT's sensitivity advantage. Pintacuda and co-workers mitigated such loss in their study of paramagnetic battery materials ${ }^{36}$ by incorporating broadbanded SHAPs ${ }^{35}$ into the $5 \pi$-pulse MAT experiment of $\mathrm{Hu}$ and coworkers. ${ }^{43}$ Note that a sixth adiabatic pulse is needed to properly refocus the $\mathrm{X}$ magnetization when using adiabatic pulses. ${ }^{44}$ Accordingly, the $t_{1}$ evolution period consists of six time intervals separated by SHAPs, timed such that the rotor phases at the center of each second SHAP are incremented by $120^{\circ}$. The $2 \mathrm{D}$ dataset is measured by incrementing timings of the second, fourth and sixth SHAPs by $t_{1} / 6$ to alter the time spent by X nuclei with +1 and -1 coherence orders, while the timings of the remaining SHAPs are unchanged (see Figure 1c). This MAT sequence can also be applied in an HMQC experiment if it is z-filtered (Figure 1c). As can 
also be seen in Figure 1c, symmetric coherence transfer pathways are selected which enables the acquisition of an amplitude modulated signal and purely absorptive-phase 2D lineshapes. ${ }^{45,46}$ This coherence transfer pathway is selected by nesting the COG3 $(2,1 ; 0)$ and COG14(0,13,0,13,0,13,0,13;7) cogwheel phase cycles ${ }^{41,42}$ in the ${ }^{1} \mathrm{H}$ and $\mathrm{X}$ channels, respectively, for a complete phase cycle lasting only 42 steps. CPMG-like coherence transfer pathways are known to be far better managed by cogwheel phase cycling; ${ }^{47}$ an equivalent nested phase cycle would have required 512 steps.

(a) CT-D-HMQC
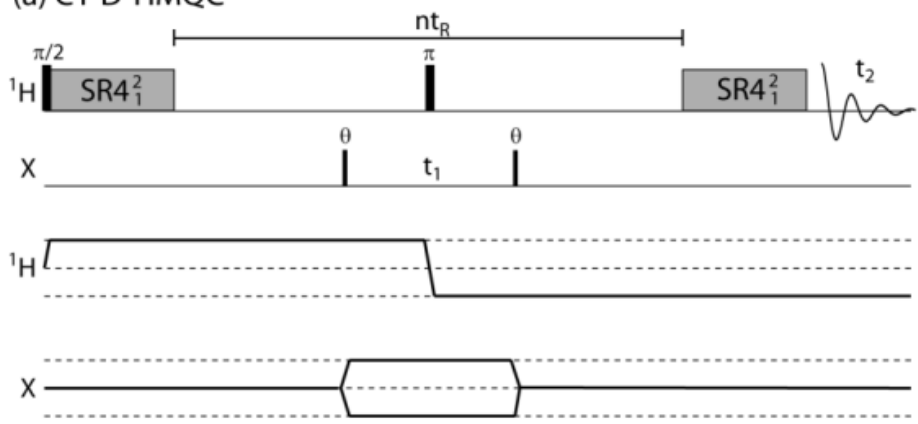

(b) D-HMQC-MAT
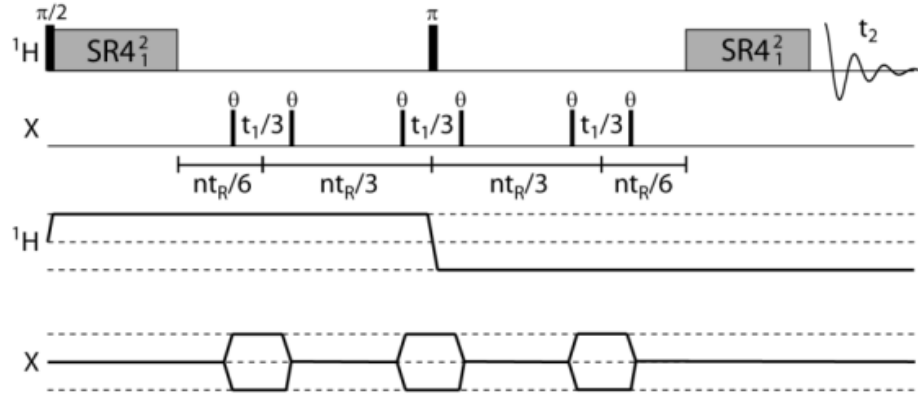

(c) D-HMQC-aMAT
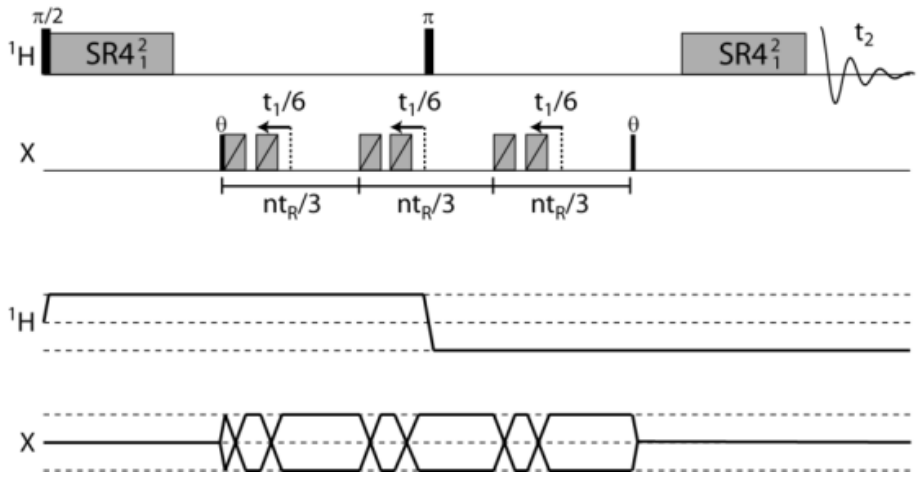
Figure 1. Pulse sequence diagrams and coherence transfer pathways for CT-D-HMQC (a), DHMQC-MAT (b), and D-HMQC-aMAT (c). Hard pulses are shown in black with the tip angle of the pulse indicated above while, in (c), "dashed" grey pulses represent adiabatic tanh/tan pulses.

\section{Sensitivity}

We first compared the sensitivity and efficacy of the HMQC methods depicted in Figure 1 by performing ${ }^{1} \mathrm{H}\left\{{ }^{195} \mathrm{Pt}\right\}$ experiments on the cisplatin and transplatin samples used earlier by Rossini and coworkers. ${ }^{24}$ In $1 \mathrm{D}{ }^{1} \mathrm{H}$-detected SSNMR experiments on cisplatin, the simplest pulse sequence (CT-D-HMQC) provides the highest sensitivity, as expected, with the D-HMQCMAT and D-HMQC-aMAT yielding 6\% and 52\% of this intensity, respectively (see Figure 2). The reduced sensitivity of the 1D D-HMQC-MAT and D-HMQC-aMAT experiments as compared to CT-D-HMQC likely reflects the limited excitation bandwidths/imperfect refocusing of the additional pulses required by the MAT portion of the experiment (vide infra). For example, with our limited rf power, the use of $50^{\circ}{ }^{195} \mathrm{Pt}$ pulses, rather than $90^{\circ}$ pulses, yielded the optimal sensitivity in the D-HMQC MAT experiment and $278 \mathrm{kHz}$ SHAPs were in fact insufficient to completely refocus the broad ${ }^{195} \mathrm{Pt}$ powder pattern.

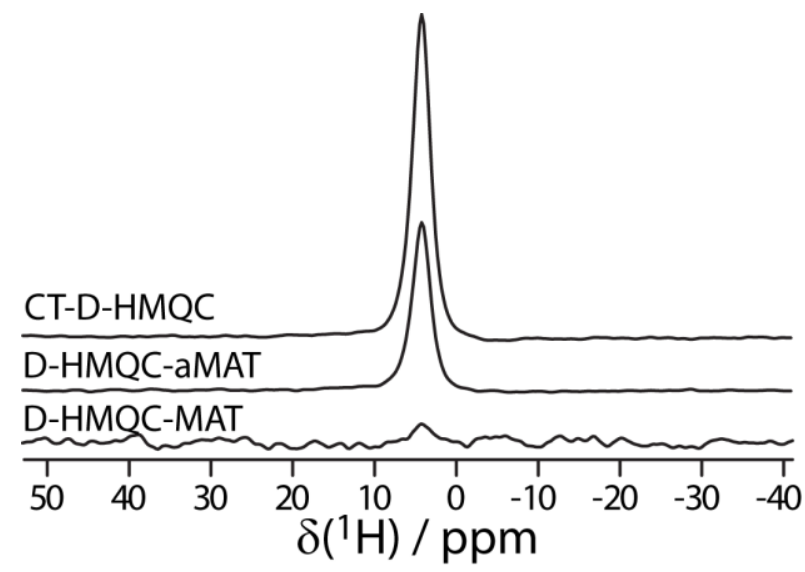

Figure 2. Comparison of the sensitivity of the first slice of the three ${ }^{1} \mathrm{H}\left\{{ }^{195} \mathrm{Pt}\right\} \mathrm{D}-\mathrm{HMQC}$ experiments depicted in Figure 1 using cisplatin as a reference. The intensity ratio between CTD-HMQC, D-HMQC-aMAT and D-HMQC-MAT is 1.00:0.52:0.06. 
The benefits of MAT-based schemes, however, are only expected in the acquisition of 2D spectra, where the folding of the spinning sidebands can lead to considerable sensitivity and resolution gains. The $2 \mathrm{D}$ spectra, and ${ }^{195} \mathrm{Pt}$ projections, of cis- and transplatin are shown in Figure 3. First, both D-HMQC-aMAT and D-HMQC-MAT experiments offer the resolution advantage of infinite-speed MAS spectra. Additionally, in spite of the losses in integrated intensity, the peak intensities compare favorably with the centerbands of the CT-D-HMQC spectra since the spectral intensity is concentrated into a single peak instead of being distributed into $\sim 15$ spinning sidebands (at $v_{\mathrm{R}}=50 \mathrm{kHz}$ ). Indeed, the intensity of the centerband is $140 \%$ and $65 \%$ higher when using the D-HMQC-aMAT and D-HMQC-MAT experiments for cisplatin, while it is $122 \%$ higher and $30 \%$ lower in the case of transplatin, for the same respective sequences (see Figure 3). If we similarly compare the intensity of the MAT peak with that of the highest-intensity spinning sideband from the CT-D-HMQC experiment we note that the intensity is $25 \%$ and $64 \%$ lower when using the D-HMQC-aMAT and D-HMQC-MAT experiments, respectively for cisplatin and $12 \%$ and $72 \%$ lower in the case of transplatin.

The apparent discrepancy between the sensitivity losses in D-HMQC-aMAT spectra in Figures 2 and 3 stems from the application of SHAPs of lengths similar to the rotor period, which leads to a slight resonance broadening (and thus reduced maximum intensity) and the introduction of spinning sidebands at $\pm 0.5 v_{R}$ and $\pm v_{R}$. These spinning-sidebands are reintroduced since the spins may not be fully refocused at the proper MAT timing when a swept pulse is used. Note, however, that in amorphous materials this sensitivity loss will be negated by the broader isotropic resonances and the D-HMQC-aMAT experiment is expected to offer a much higher sensitivity, vide infra. SIMPSON simulations ${ }^{48,49}$ show that longer SHAPs can be used to eliminate these artifacts; however, in our case, these would significantly lengthen the echo time 
and increase the reflected power. We found that the SHAPS lasting $1.5 t_{\mathrm{R}}$ are a good compromise to reduce this CSA-recoupling and obtain high-quality spectra. In the subsequent sections, the D-HMQC-aMAT experiment will be applied to analyze a decomposed sample of transplatin as well as a Pt-impregnated metal-organic framework (MOF): $\mathrm{UiO}-66-\mathrm{NH}_{2}$, both of which contain multiple ${ }^{195} \mathrm{Pt}$ resonances that would have been difficult to analyze otherwise. 
(a) cis-platin

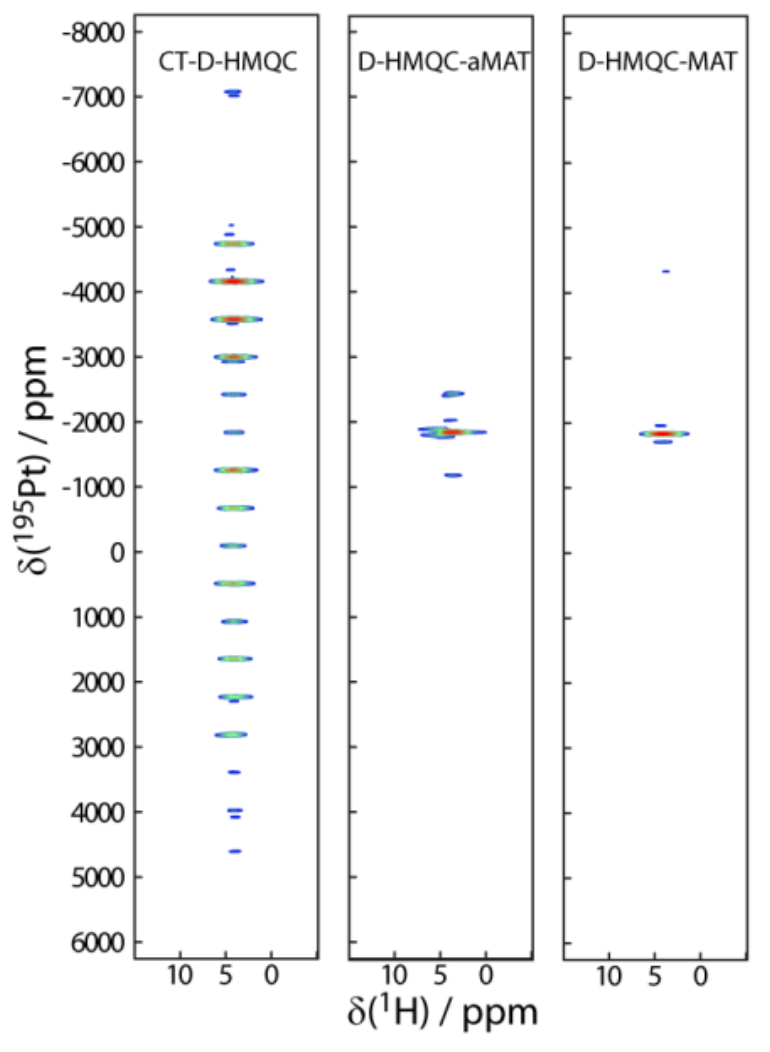

(b) trans-platin

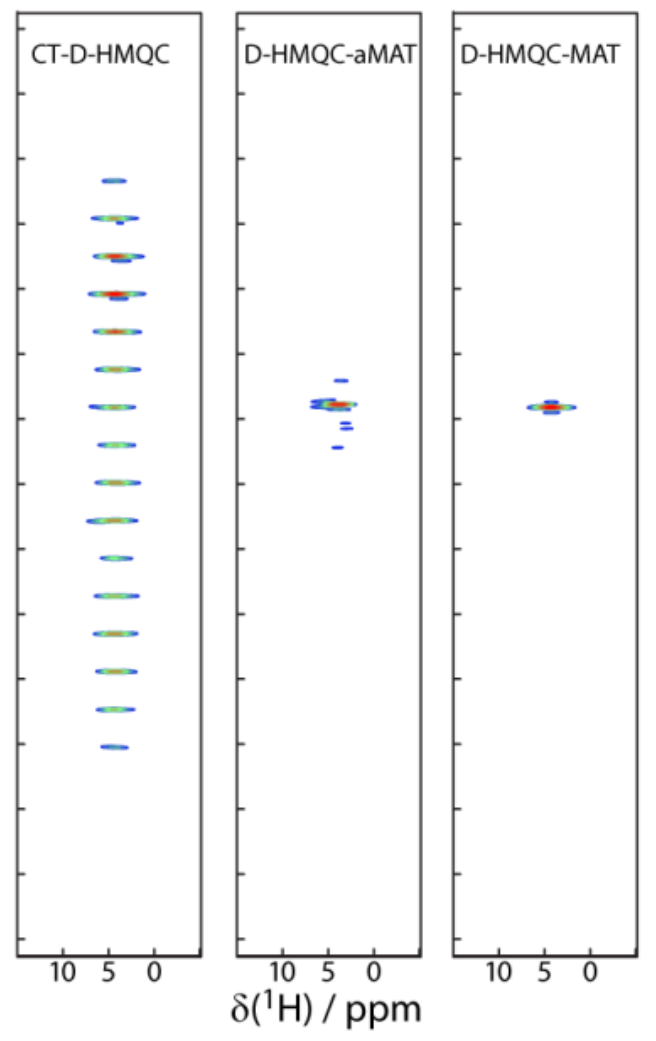

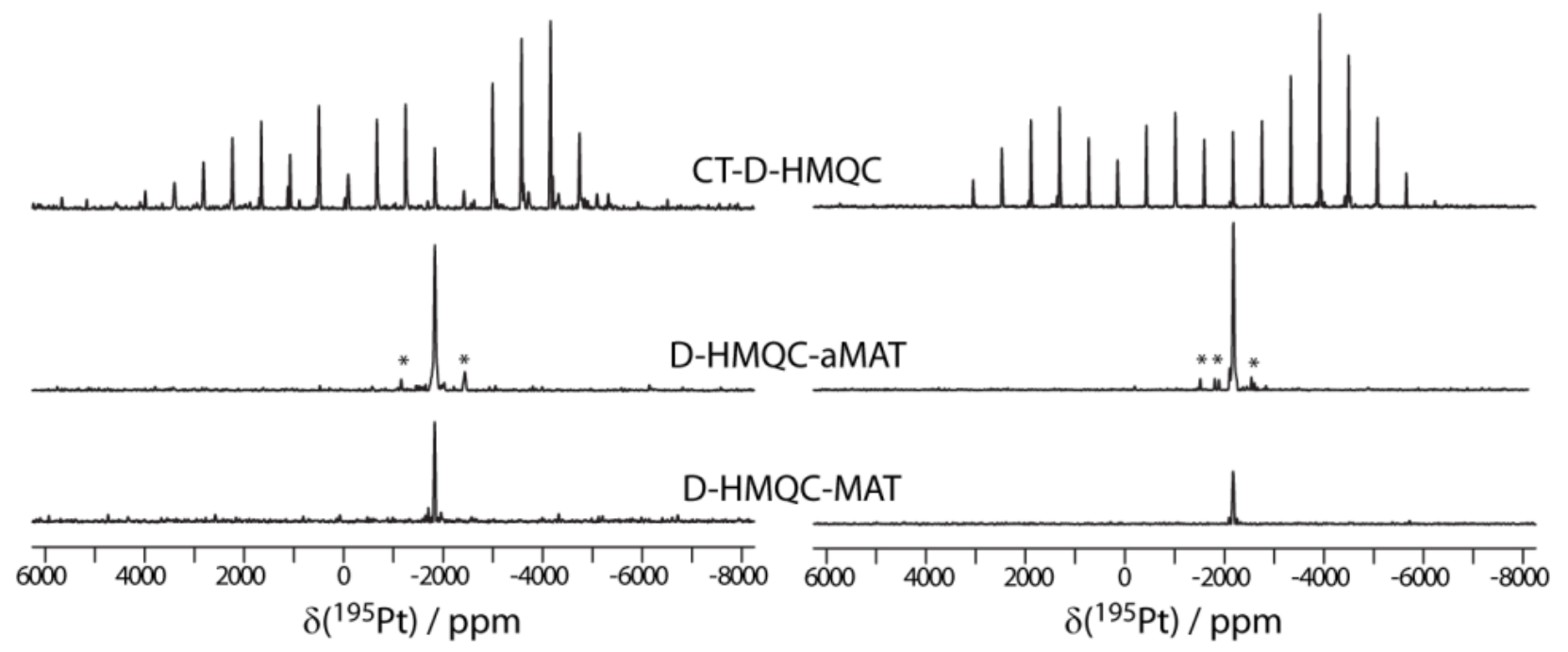

Figure 3. Comparison of the 2D CT-D-HMQC, D-HMQC-aMAT and D-HMQC-MAT spectra of cisplatin (a) and transplatin (b) taken using $v_{\mathrm{R}}=50 \mathrm{kHz}$. The ${ }^{195} \mathrm{Pt}$ projections of these spectra, corrected for the number of scans, are shown at the bottom. Asterisks in the 1D projections denote residual spinning sidebands. 


\section{Decomposed Transplatin}

An impure (decomposed) sample of transplatin, purchased from Sigma Aldrich, has been previously described by Rossini and coworkers. ${ }^{24}$ The 2D CT-D-HMQC and D-HMQC-aMAT spectra of this sample are shown in Figure 4 along with their ${ }^{195} \mathrm{Pt}$ projections. Three different sideband manifolds, similar to those of transplatin, can be distinguished in the CT-D-HMQC spectra along with an intense, sideband-less resonance at $-410 \mathrm{ppm}$ assigned to an octahedral $\mathrm{Pt}(\mathrm{IV})$ center. The CT-D-HMQC experiment has a relatively low sensitivity in this case, due in part to a larger number of resonances in the spectrum and their broad linewidths. The sensitivity and resolution are, however, greatly improved in the D-HMQC-aMAT spectrum, which also no longer overemphasizes the Pt(IV) site with near-zero CSA, when compared to the three isotropic resonances from the $\mathrm{Pt}(\mathrm{II})$ sites at $-2070,-1920$, and $-1770 \mathrm{ppm}$. The improved resolution also allowed us to detect a previously unnoticed resonance at $130 \mathrm{ppm}$. Importantly, while the maximum intensity of the $\mathrm{Pt}(\mathrm{IV})$ resonance decreased by $26 \%$ in the D-HMQC-aMAT experiment, those of the $\mathrm{Pt}(\mathrm{II})$ sites dramatically increased by $450 \%$ and $260 \%$ when compared to the centerband and highest intensity sideband, respectively. Therefore, as previously stated, great sensitivity gains are to be expected when performing the aMAT experiment in $t_{1}$ in situations where the isotropic linewidths are broad. Note that the SHAPs are most effective for the $\mathrm{Pt}(\mathrm{IV})$ site and thus the spectrum is not quantitative. Contrary to this, however, if we assume identical recoupling efficiencies, the D-HMQC-MAT experiment is expected to be quantitative. 
(a)

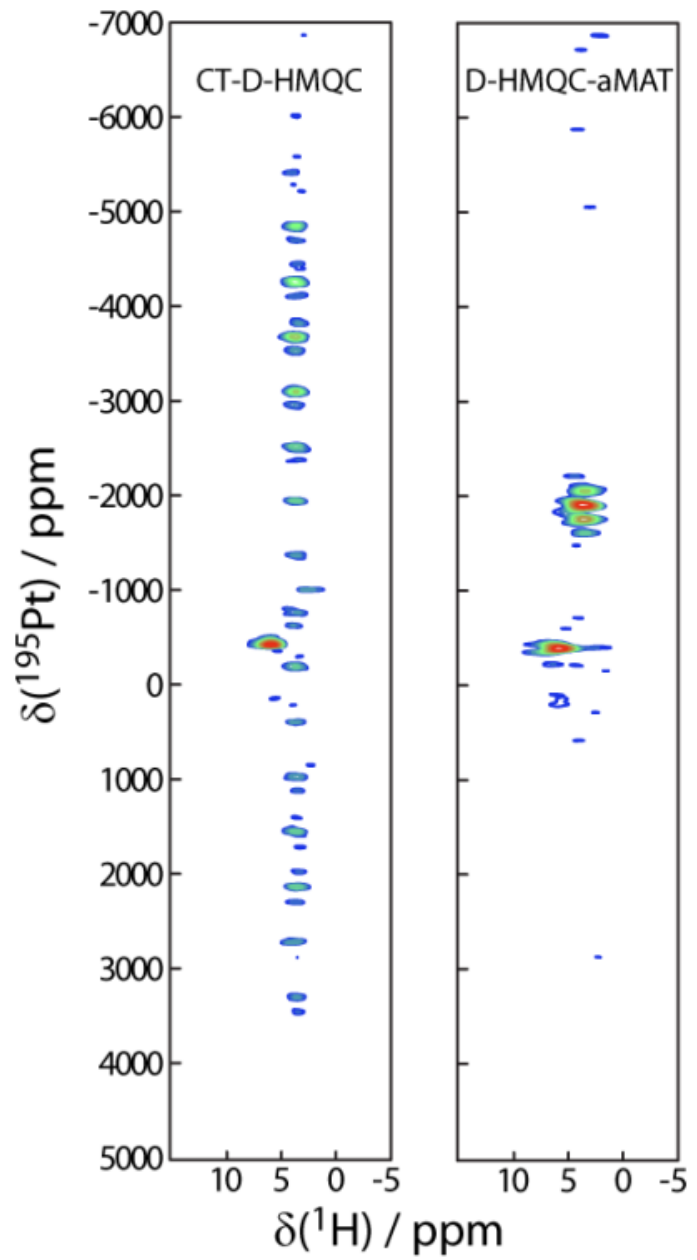

(b)

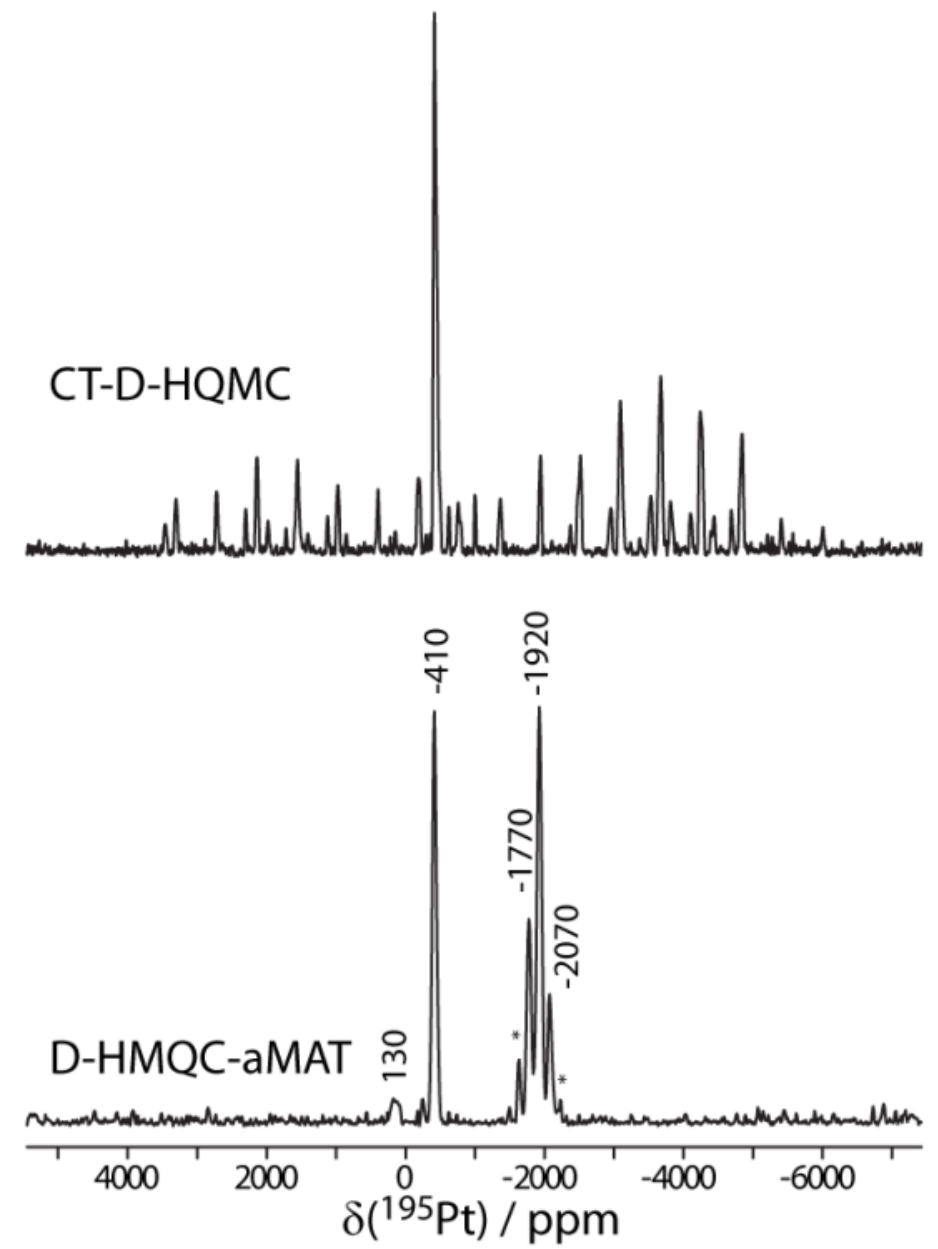

Figure 4. (a) 2D CT-D-HMQC and D-HMQC-aMAT spectra of decomposed transplatin. As demonstrated by the corresponding ${ }^{195} \mathrm{Pt}$ projections (b), the use of MAT greatly improves the resolution and sensitivity thus enabling the detection five isotropic peaks including a previouslyundetected $\mathrm{Pt}(\mathrm{IV})$ resonance at $130 \mathrm{ppm}$. Residual spinning sidebands at $\pm 0.5 v_{\mathrm{R}}$ are marked by asterisks in (b).

The exceptional sensitivity of the ${ }^{195} \mathrm{Pt}$ chemical shifts to platinum's first coordination sphere allows us to propose a simple decomposition pathway. If we assume that transplatin had decomposed through a solid-phase hydrolysis reaction, only complexes with permutations of $\mathrm{NH}_{3}, \mathrm{Cl}^{-}$, and $\mathrm{OH}^{-}\left(\right.$or $\mathrm{H}_{2} \mathrm{O}$ ) as ligands are possible. ${ }^{50,51}$ Because any ligand substitution, within this family, affects the ${ }^{195} \mathrm{Pt}$ chemical shift by $150-450 \mathrm{ppm},{ }^{50,51}$ only the proposed mechanism in 
Scheme 1 can explain the observed ${ }^{195} \mathrm{Pt}$ chemical shifts. We assign the resonance at $-2070 \mathrm{ppm}$ to residual, amorphous, transplatin while the two other Pt(II) peaks correspond to the first hydrolysis products wherein a chloride was replaced by a hydroxyl moiety. The recentlyreleased chloride anion then coordinates to $\mathrm{Pt}(\mathrm{IV})$ centers, that have been oxidized by water, to produce $\left[\mathrm{Pt}\left(\mathrm{NH}_{3}\right)_{3} \mathrm{Cl}_{3}\right]^{+}$and $\left[\mathrm{PtCl}_{6}\right]^{2-}$ complexes represented by the resonances at -410 and 130 ppm, respectively. These complexes also feature an increased amine ${ }^{1} \mathrm{H}$ chemical shift of $6 \mathrm{ppm}$ in agreement with the increased Pt oxidation state. ${ }^{50}$ The amine protons resonate at 4 ppm when coordinated to a $\mathrm{Pt}(\mathrm{II})$ site. The resonance assigned to $\left[\mathrm{PtCl}_{6}\right]^{2-}$ is significantly weaker due to its lack of protonated ligands.

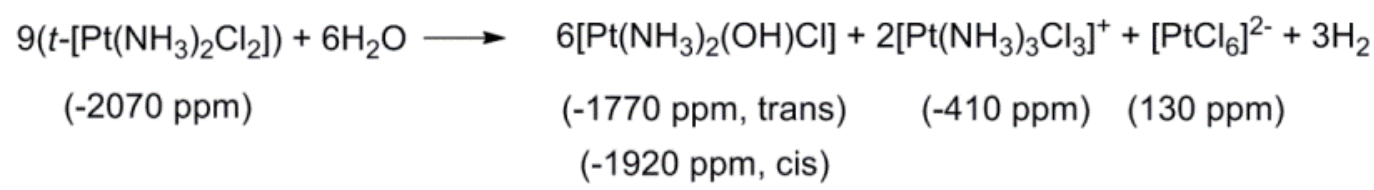

Scheme 1. Proposed solid-phase hydrolysis decomposition reaction of transplatin and proposed isotropic chemical shift assignment, supported by high-resolution ${ }^{195} \mathrm{Pt}$ SSNMR spectroscopy.

\section{Pt-loaded UiO-66- $\mathrm{NH}_{2}$}

Recently, we have studied platinum species supported inside various MOFs with the use of DNP-enhanced ${ }^{15} \mathrm{~N}$ MAS ${ }^{52}$ as well as ${ }^{195} \mathrm{Pt}$ wideline SSNMR. ${ }^{13}$ Of particular focus was the interaction between the $\mathrm{Pt}(\mathrm{II})$ ions and the amine-modified UiO-66 MOF (denoted as $\mathrm{Pt}(\mathrm{II}) / \mathrm{UiO}-$ 66- $\mathrm{NH}_{2}$ ). The ${ }^{15} \mathrm{~N}$ SSNMR spectra allowed us to monitor the loading of Pt inside the MOF and detail its coordination environment near the amine functional groups of the linkers. Ultrawideline, DNP-enhanced, BRAIN-CP-WCPMG experiments enabled the distinction, and quantification, of the cis- and trans-coordinated platinum conformers (see Figure 5) by their CSA and allowed us to comment on their relative reactivity. Under static conditions, however, DNP was only able to yield sensitivity improvements of $\sim 6$, which is very similar to what can be, in 
principle, achieved by indirect detection. The use of the methods presented here could then have a further impact on the resolution and sensitivity of ${ }^{195} \mathrm{Pt}$ SSNMR of Pt-loaded MOFs.

We have performed D-HMQC-aMAT and CT-D-HMQC experiments on a Pt(II)/UiO66- $\mathrm{NH}_{2} \mathrm{MOF}$ with $20 \%$ w/w of platinum. A high quality ( $\left.\mathrm{S} / \mathrm{N}=12.5\right)$ D-HMQC-aMAT spectrum could be obtained in only 5.2 hours, revealing the presence of two $\operatorname{Pt}(\mathrm{II})$ environments with chemical shifts of -2030 and $-1720 \mathrm{ppm}$ (Figure 5). The peak centered at $-2030 \mathrm{ppm}$ can be straightforwardly assigned to the cis- and trans- $\left[\mathrm{PtN}_{2} \mathrm{Cl}_{2}\right]$ coordination modes, which were previously identified at around $-1983 \mathrm{ppm}$ and $-2083 \mathrm{ppm}$, respectively, ${ }^{13}$ but here remained unresolved. This lack of resolution is most likely caused by the similarity between the two sites ${ }^{50}$ and the dispersions of their isotropic chemical shifts. The less intense peak at $-1720 \mathrm{ppm}$ represents a hydrolysis product of the form $\left[\mathrm{PtN}_{2}(\mathrm{OH}) \mathrm{Cl}\right]$, see Figure $5 .{ }^{50}$ The hydrolysis product was not detected by ultra-wideline ${ }^{195} \mathrm{Pt}$ SSNMR since it likely has very similar CSA as the parent complex and is found in a much lower quantity. The $\left[\mathrm{PtN}_{2} \mathrm{Cl}_{2}\right]$ site is correlated to a ${ }^{1} \mathrm{H}$ chemical shift of $8.5 \mathrm{ppm}$ while the $\left[\mathrm{PtN}_{2}(\mathrm{OH}) \mathrm{Cl}\right]$ site is correlated to a ${ }^{1} \mathrm{H}$ chemical shift of 7.8 $\mathrm{ppm}$. The lower chemical shift, in the case of the hydrolyzed product, is caused by the hydroxyl's lower chemical shifts. Both sites have unusually high ${ }^{1} \mathrm{H}$ chemical shifts due to hydrogen bonding interactions with adsorbed water.

This example demonstrates that the use of D-HMQC-aMAT in concert with ultrawideline SSNMR yields a more complete picture of the platinum environments in the $\mathrm{Pt}(\mathrm{II}) / \mathrm{UiO}-$ 66- $\mathrm{NH}_{2} \mathrm{MOF}$, that neither technique alone could provide. Like the previous example, the DHMQC-aMAT experiment again offers a great sensitivity advantage over the CT-D-HMQC experiment since the additional broadening from the SHAPs only has a minor impact on the broad isotropic resonances. We estimate that the D-HMQC-aMAT centerband is approximately 
three times more intense than the highest intensity sideband in the low-sensitivity CT-D-HMQC spectrum (not shown). This estimate is also in agreement with the $\sim 50 \%$ efficiency of the aMAT experiment on our spectrometer, as depicted in Figure 2. In order to obtain a CT-D-HMQC experiment of similar quality, the experiment would then require at least two days of continuous acquisition, although an even longer experiment time would be required to accurately measure the components of the chemical shift tensor.

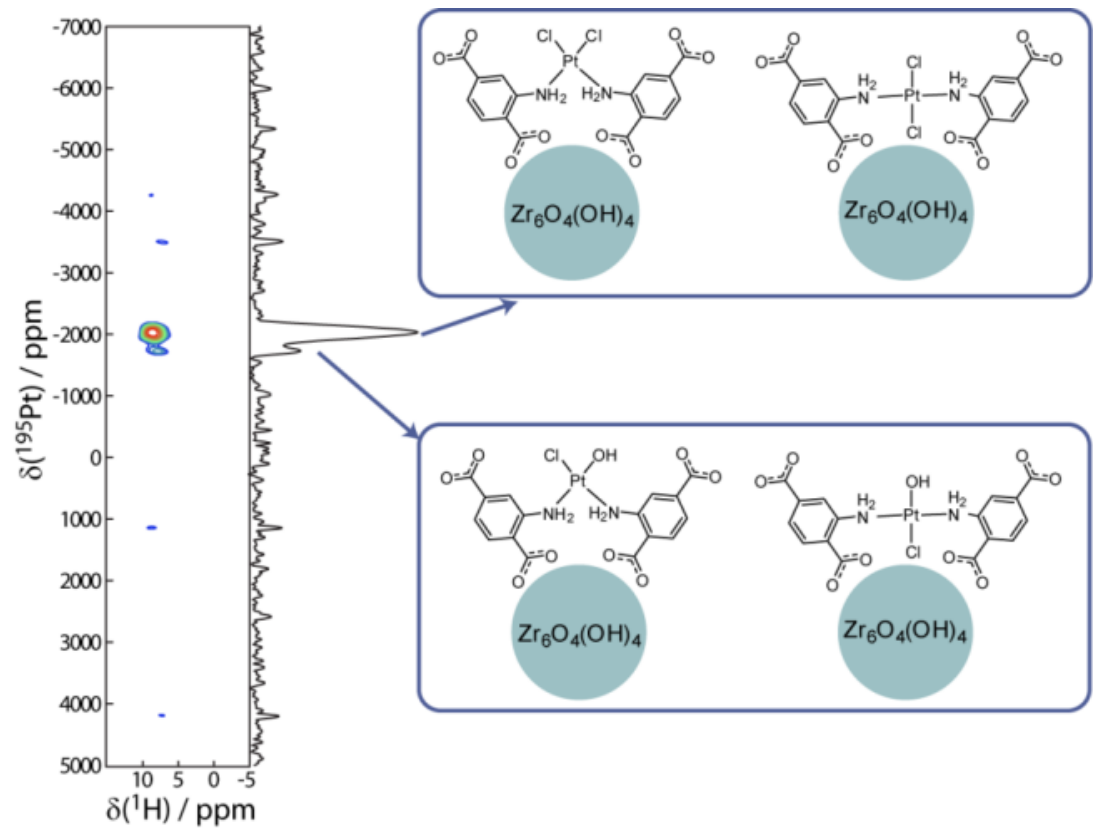

Figure 5. The D-HMQC-aMAT spectrum of $\mathrm{Pt}(\mathrm{II}) / \mathrm{UiO}-66-\mathrm{NH}_{2} \mathrm{MOF}$ featuring cis and trans $\left[\mathrm{PtN}_{2} \mathrm{Cl}_{2}\right]$ coordination modes (top right) and the previously undetected hydrolysis products $\left[\mathrm{PtN}_{2}(\mathrm{OH}) \mathrm{Cl}\right]$ (bottom right).

\section{Conclusions}

We have reported two new approaches, D-HMQC-MAT and D-HMQC-aMAT, for measuring infinite-speed MAS SSNMR spectra of heavy spin-1/2 nuclides that typically yield ultra-wide spectra. The experiments use (i) a broadbanded-MAT sequence in the indirect dimension of a constant time D-HMQC experiment, which enables the folding of the spinning sidebands into the centerband while allowing the use of arbitrary (i.e., non-rotor-synchronized) 
increments of $t_{1}$ for better control of the indirect spectral widths, and (ii) indirect detection by the ${ }^{1} \mathrm{H}$ spins to maximize the sensitivity and increase resolution. The D-HMQC-aMAT scheme, in particular, provides the most sensitive technique to date for the measurement of high-resolution spectra of these problematic nuclides.

Notably, we have shown that the D-HMQC-aMAT experiment allowed for the resolution of five distinct Pt environments in a decomposed transplatin sample, thus allowing us to propose a decomposition mechanism. We have additionally shown that, using this method, a highresolution MAS ${ }^{195} \mathrm{Pt}$ SSNMR spectrum can be obtained for a Pt-impregnated MOF in a similar amount of time as was needed to acquire an ultra-wideline static SSNMR spectrum using DNP. The sensitivity of the infinite-speed MAS experiments presented here can be further improved with the application of DNP. We expect that these experiments will complement ultra-wideline SSNMR methods, both static and MAS-based, such that the isotropic chemical shifts as well as the CSA tensor components can be measured to determine coordination of the challenging heavy spin-1/2 nuclides in a wide range of materials.

\section{Experimental}

\section{Materials}

Cis- and transplatin were purchased from Alfa Aesar and used as received while a "decomposed" transplatin sample was obtained from Sigma Aldrich. The platinum-impregnated UiO-66- $\mathrm{NH}_{2} \mathrm{MOF}$ was prepared as previously described. ${ }^{52}$

SSNMR

All experiments were performed on a Bruker AVANCE III HD 400 MHz SSNMR spectrometer equipped with a double resonance Bruker 1.3-mm probe capable of MAS at frequencies up to $67 \mathrm{kHz}$. The samples were tightly packed into 1.3 -mm zirconia rotors and 
spun at $50 \mathrm{kHz}$ for all experiments. The ${ }^{1} \mathrm{H} \pi / 2$ and $\pi$ pulses lasted 2.5 and $5 \mu$ s, respectively and the SR $4_{1}^{2}$ recoupling scheme, lasting between 84 and 108 rotor periods, was used. The central echo time was typically set to 10-20 rotor periods. In the case of the CT-D-HMQC and DHMQC-aMAT experiments, the ${ }^{195} \mathrm{Pt}$ square pulses lasted $0.6 \mu$ s with an rf power of ca. $278 \mathrm{kHz}$ ( $60^{\circ}$ tip angle) while in the case of the D-HMQC-MAT experiments, a $0.5 \mu$ s pulse yielded the best sensitivity. The SHAPs used were tanh/tan pulses sweeping over $5 \mathrm{MHz}$ in either 20 or 30 $\mu$ sith rf powers of 278 and $260 \mathrm{kHz}$, respectively. In all cases, the recycle delay was set to $1 \mathrm{~s}$ with the exception of UiO-66- $\mathrm{NH}_{2}$ where it was set to $0.5 \mathrm{~s} .32$ to 320 scans were accumulated for each of the $512 t_{1}$ increments of $0.8 \mu \mathrm{s}$ ( 128 in the case of UiO-66- $\mathrm{NH}_{2}$ ) and the States-TPPI method was used for phase-sensitive detection.

\section{Acknowledgements}

The authors thank Dr. T. Kobayashi for helpful discussions. This research is supported by the U.S. Department of Energy, Basic Energy Sciences, Division of Chemical Sciences, Geosciences, and Biosciences through the Ames Laboratory. Ames Laboratory is operated for the DOE by Iowa State University under Contract No. DEAC02-07CH11358. A.J.R. and W.H. gratefully acknowledge startup fund support from the Ames Laboratory (Royalty Account) and Iowa State University. Partial support for F.P. is through a Spedding Fellowship funded by the Laboratory Directed Research and Development (LDRD) program at the Ames Laboratory. F. P. thanks NSERC (Natural Sciences and Engineering Research Council of Canada) and the Government of Canada for a Banting Postdoctoral Fellowship. 


\section{References}

${ }^{1}$ R. W. Schurko, Ultra-wideline solid-state NMR spectroscopy, Acc. Chem. Res. 46 (2013) 1985-1995.

${ }^{2}$ D. Massiot, I. Farnan, N. Gautier, D. Trumeau, A. Trokiner, J. P. Coutures, ${ }^{71} \mathrm{Ga}$ and ${ }^{69} \mathrm{Ga}$ nuclear magnetic resonance study of $\beta-\mathrm{Ga}_{2} \mathrm{O}_{3}$ : resolution of four- and six-fold coordinated Ga sites in static conditions, Solid State Nucl. Magn. Reson. 4 (1995) 241-248.

${ }^{3}$ J. T. Cheng, P. D. Ellis, Adsorption of $\mathrm{Rb}+$ to $\gamma$-alumina followed by solid-state ${ }^{87} \mathrm{Rb} \mathrm{NMR}$ spectroscopy, J. Phys. Chem. 93 (1989) 2549-2555.

${ }^{4}$ S. S. Berr, R. R. M. Jones, Small-angle neutron scattering from acqueous solutions of sodium perfluorooctanoate above the critical micelle concentration, J. Phys. Chem. 93 (1989) 25552558.

${ }^{5}$ F. H. Larsen, H. J. Jakobsen, P. D. Ellis, N. C. Nielsen, Sensitivity-enhanced quadrupolar-echo NMR of half-integer quadrupolar nuclei. Magnitudes and relative orientation of chemical shielding and quadrupolar coupling tensors, J. Phys. Chem. A 101 (1997) 8597-8606.

${ }^{6}$ F. H. Larsen, J. Skibsted, H. J. Jakobsen, N. C. Nielsen, Solid-state QCPMG NMR of low- $\gamma$ quadrupolar metal nuclei in natural abundance, J. Am. Chem. Soc. 122 (2000) 7080-7086.

${ }^{7}$ A. Medek, V. Frydman, L. Frydman, Central transition nuclear magnetic resonance in the presence of large quadrupole couplings: cobalt-59 nuclear magnetic resonance of cobaltophtalocyanines, J. Phys. Chem A 103 (1999) 4830-4835.

${ }^{8}$ J. Persons, G. S. Harbison, Slow turning reveals enormous quadrupolar interactions (STREAQI), J. Magn. Reson. 186 (2007) 347-351.

${ }^{9}$ R. Bhattacharyya, L. Frydman, Quadrupolar nuclear magnetic resonance spectroscopy in solids using frequency-swept echoing pulses, J. Chem. Phys. 127 (2007) 194503. 
${ }^{10} \bar{E}$. Kupče, R. Freeman, Adiabatic pulses for wideband inversion and broadband decoupling, J. Magn. Reson. Ser. A 115 (1995) 273-276.

${ }^{11}$ L. A. O’Dell, R. W. Schurko, QCPMG using adiabatic pulses for faster acquisition of ultrawideline NMR spectra, Chem. Phys. Lett. 464 (2008) 97-102.

${ }^{12}$ K. J. Harris, A. Lupulescu, B. E. G. Lucier, L. Frydman, R. W. Schurko, Broadband adiabatic inverstion pulses for cross-polarization in wideline solid-state NMR spectroscopy, J. Magn. Reson. 2012, 224, 38-47.

${ }^{13}$ T. Kobayashi, F. A. Perras, T. W. Goh, T. L. Metz, W. Huang, M. Pruski, DNP-enhanced ultrawideline solid-state NMR spectroscopy: studies of platinum in metal-organic frameworks, J. Phys. Chem. Lett. 7 (2016) 2322-2327.

${ }^{14}$ D. A. Hirsh, A. J. Rossini, L. Emsley, R. W. Schurko, ${ }^{35} \mathrm{Cl}$ dynamic nuclear polarization solidstate NMR of active pharmaceutical ingredients, Phys. Chem. Chem. Phys. 18 (2016) 2589325904.

${ }^{15}$ Y. Nishiyama, Fast magic-angle sample spinning solid-state NMR at $60-100 \mathrm{kHz}$ for natural abundance samples, Solid State Nucl. Magn. Reson. 78 (2016) 24-36.

${ }^{16}$ A.-C. Pöppler, J. P. Demers, M. Malon, A. P. Singh, H. W. Roesky, Y. Nishiyama, A. Lange, Ultrafast magic-angle spinning: benefits for the acquisition of ultrawide-line NMR spectra of heavy spin-1/2 nuclei, ChemPhysChem 17 (2016) 812-816.

${ }^{17}$ K. Yamauchi, J. W. G. Janssen, A. P. M. Kentgens, Implementing solenoid microcoils for wide-line solid-state NMR, J. Magn. Reson. 167 (2004) 87-96.

${ }^{18}$ A. P. M. Kentgens, J. Bart, P. J. M. van Bentum, A. Brinkmann, E. R. H. van Eck, J. G. E. Gardeniers, J. W. G. Janssen, P. Knijn, S. Vasa, M. H. W. Verkuijlen, High-resolution liquid- 
and solid-state nuclear magnetic resonance of nanoliter sample volumes using microcoil detectors, J. Chem. Phys. 128 (2008) 052202.

${ }^{19}$ J. A. Tang, L. A. O’Dell, P. M. Aguiar, B. E. G. Lucier, D. Sakellariou, R. W. Schurko, Application of static microcoils and WURST pulses for solid-state ultra-wideline NMR spectroscopy of quadrupolar nuclei, Chem. Phys. Lett. 466 (2008) 227-234.

${ }^{20}$ Y.-Y. Hu, E. M. Levin, K. Schmidt-Rohr, Broadband "infinite-speed" magic-angle spinning NMR spectroscopy, J. Am. Chem. Soc. 131 (2009) 8390-8391

21 Y.-Y. Hu, K. Schmidt-Rohr, Technical aspects of fast magic-angle turning NMR for dilute spin-1/2 nuclei with broad spectra, Solid State Nucl. Magn. Reson. 40 (2011) 51-59.

${ }^{22}$ Z. Gan, High-resolution chemical shift and chemical shift anisotropy correlation in solids using slow magic angle spinning, J. Am. Chem. Soc. 114 (1992) 8307-8309.

${ }^{23}$ I. Hung, L. Zhou, F. Pourpoint, C. P. Grey, Z. Gan, Isotropic high field NMR spectra of Li-ion battery materials with anisotropy >1 MHz, J. Am. Chem. Soc. 134 (2012) 1898-1901.

${ }^{24}$ A. J. Rossini, M. P. Hanrahan, M. Thuo, Rapid acquisition of wideline MAS solid-state NMR spectra with fast MAS, proton detection, and dipolar HMQC pulse sequences, Phys. Chem. Chem. Phys. 18 (2016) 25284-25295.

${ }^{25}$ Y. Ishii, R. Tycko, Sensitivity enhancement in solid state ${ }^{15} \mathrm{~N}$ NMR by indirect detection with high-speed magic angle spinning, J. Magn. Reson. 142 (2000) 199-204.

${ }^{26}$ Y. Ishii, J. P. Yesinowski, R. Tycko, Sensitivity enhancement in solid-state ${ }^{13} \mathrm{C}$ NMR of synthetic polymers and biopolymers by ${ }^{1} \mathrm{H}$ NMR detection with high-speed magic angle spinning, J. Am. Chem. Soc. 123 (2001) 2921-2922. 
${ }^{27}$ J. W. Wiench, C. E. Bronnimann, V. S.-Y. Lin, M. Pruski, Chemical shift correlation NMR spectroscopy with indirect detection in fast rotating solids: studies of organically functionalized mesoporous silicas, J. Am. Chem. Soc. 129 (2007) 12076-12077.

${ }^{28}$ D. H. Zhou, G. Shah, M. Cormos, C. Mullen, D. Sandoz, C. M. Rienstra, Proton-detected solid-state NMR spectroscopy of fully protonated proteins at $40 \mathrm{kHz}$ magic-angle spinning, $\mathrm{J}$. Am. Chem. Soc. 129 (2007) 11791-11801.

${ }^{29} \mathrm{Z}$. Gan, ${ }^{13} \mathrm{C} /{ }^{14} \mathrm{~N}$ heteronuclear multiple-quantum correlation with rotary resonance and REDOR dipolar recoupling, J. Magn. Reson. 184 (2007) 39-43.

${ }^{30}$ G. Tricot, J. Trébosc, F. Pourpoint, R. Gauvin, L. Delevoye, The D-HMQC MAS-NMR technique: an efficient tool for the editing of through-space correlation spectra between quadrupolar and spin-1/2 $\left({ }^{31} \mathrm{P},{ }^{29} \mathrm{Si},{ }^{1} \mathrm{H},{ }^{13} \mathrm{C}\right)$ nuclei, Annu. Rep. NMR Spectrosc. 81 (2014) 145-184.

${ }^{31}$ O. N. Antzutkin, Sideband manipulation in magic-angle-spinning nuclear magnetic resonance, Prog. Nucl. Magn. Reson. Spectrosc. 35 (1999) 203-266.

${ }^{32}$ W. T. Dixon, J. Schaefer, M. D. Sefcik, E. O. Stejskal, R. A. McKay, Total suppression of sidebands in CPMAS C-13 NMR, J. Magn. Reson. 49 (1982) 341-345.

${ }^{33}$ W. T. Dixon, Spinning-sideband-free and spinning-sideband-only NMR spectra in spinning samples, J. Chem. Phys. 77 (1982) 1800-1809

${ }^{34}$ J. Hong, G. S. Harbison, Magic-angle-spinning sideband elimination by temporary interruption of the chemical shift, J. Magn. Reson. Ser. A 105 (1993) 128-136.

${ }^{35}$ G. Kervern, G. Pintacuda, L. Emsley, Fast adiabatic pulses for solid-state NMR of paramagnetic systems, Chem. Phys. Lett. 435 (2007) 157-162. 
${ }^{36}$ R. J. Clément, A. J. Pell, D. S. Middlemiss, F. C. Strobridge, J. K. Miller, M. S. Whittingham, L. Emsley, C. P. Grey, G. Pintacuda, Spin-transfer pathways in paramagnetic lithium transition-metal phosphates from combined broadband isotropic solid-state MAS NMR spectroscopy and DFT calculations, J. Am. Chem. Soc. 134 (2012) 17178-17185.

${ }^{37}$ A. Bax, R. H. Griffey, B. L. Hawkins, Correlation of proton and nitrogen-15 chemical shifts by multiple quantum NMR, J. Magn. Reson. 55 (1983) 301-315.

${ }^{38}$ A. Brinkmann, A. P. Kentgens, Proton-selective ${ }^{17} \mathrm{O}-{ }^{1} \mathrm{H}$ distance measurements in fast magicangle-spinning solid-state NMR spectroscopy for the determination of hydrogen bond lengths, J. Am. Chem. Soc. 128 (2006) 14758-14759.

${ }^{39}$ B. Hu, J. Trébosc, J. P. Amoureux, Comparison of several hetero-nuclear dipolar recoupling NMR methods to be used in MAS HMQC/HSQC, J. Magn. Reson. 192 (2008) 112-122.

${ }^{40}$ N. M. Szeverenyi, A. Bax, G. E. Maciel, Magic-angle hopping as an alternative to magic-angle spinning for solid-state NMR, J. Magn. Reson. 61 (1985) 440-447.

${ }^{41}$ M. H. Levitt, P. K. Madhu, C. E. Hughes, Cogwheel phase cycling, J. Magn. Reson. 155 (2002) 300-306.

${ }^{42}$ A. Jerschow, R. Kumar, Calculation of coherence pathway selection and cogwheel cycles, J. Magn. Reson. 160 (2003) 59-64.

${ }^{43}$ J. Z. Hu, W. Wang, F. Liu, M. S. Solum, D. W. Alderman, R. J. Pugmire, D. M. Grant, Magicangle-turning experiments for measuring chemical-shift-tensor principle values in powdered solids, J. Magn. Reson. Ser A 113 (1995) 210-222.

${ }^{44}$ S. Conolly, G. Glover, D. Nishimura, A. Macovski, A reduced power selective adiabatic spinecho pulse sequence, Magn. Reson. Med. 18 (1991) 28-38. 
${ }^{45}$ O. W. Sørensen, M. Rance, R. R. Ernst, z filters for purging phase- or multiplet-distorted spectra, J. Magn. Reson. 56 (1984) 527-534.

${ }^{46}$ J.-P. Amoureux, C. Fernandez, S. Steuernagel, Z filtering in MQMAS NMR, J. Magn. Reson. Ser A 123 (1996) 116-118.

${ }^{47}$ N. Ivchenko, C. E. Hughes, M. H. Levitt, Application of cogwheel phase cycling to sideband manipulation experiments in solid-state NMR, J. Magn. Reson. 164 (2003) 286-293.

${ }^{48}$ M. Bak, J. T. Rasmussen, N. C. Nielsen, SIMPSON: A general simulation program for solidstate NMR spectroscopy, J. Magn. Reson. 147 (2000) 296-330.

${ }^{49}$ Z. Tošner, T. Vosegaard, C. Kehlet, N. Khaneja, S. J. Glaser, N. C. Nielsen, Optimal control in NMR spectroscopy: numerical implementation in SIMPSON, J. Magn. Reson. 197 (2009) 120134.

${ }^{50}$ S. J. Berners-Price, L. Ronconi, P. J. Sadler, Insights into the mechanism of action of platinum anticancer drugs from multinuclear NMR spectroscopy, Prog. Nucl. Magn. Reson. Spectrosc. 49 (2006) 65-98.

${ }^{51}$ P. S. Pregosin, Platinum NMR spectroscopy, Annu. Rep. NMR Spectrosc. 17 (1986) 285-349.

${ }^{52}$ Z. Guo, T. Kobayashi, L.-L. Wang, T. W. Goh, C. Xiao, M. A. Caporini, M. Rosay, D. D. Johnson, M. Pruski, W. Huang, Selective host-guest interaction between metal ions and metalorganic frameworks using dynamic nuclear polarization enhanced solid-state NMR spectroscopy, Chem. Eur. J. 20 (2014) 16308-16313. 


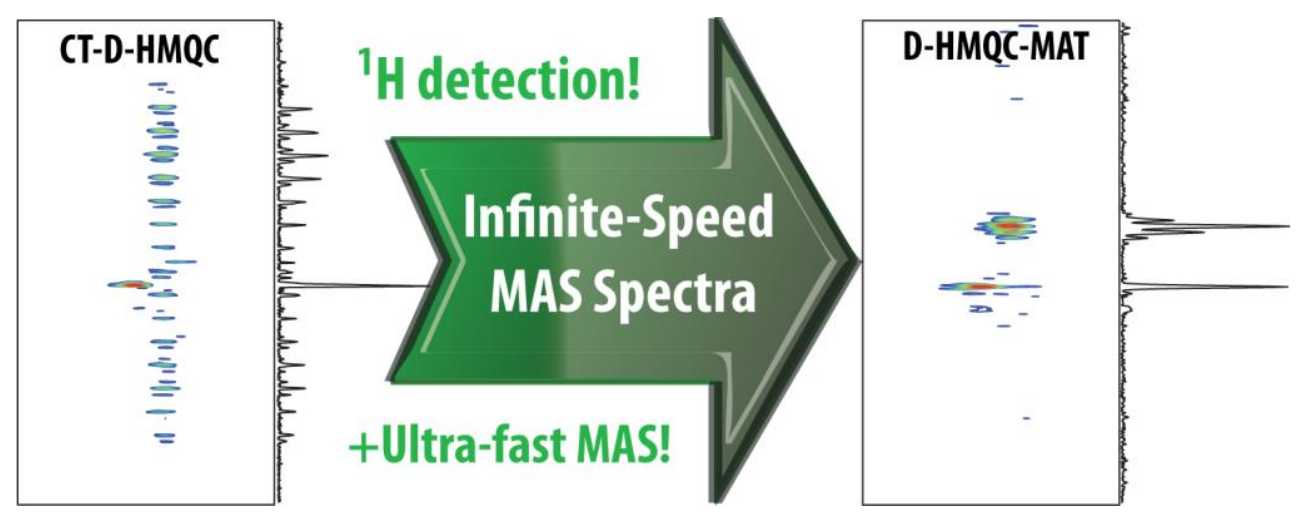

